\title{
NUEVOS DATOS SOBRE LA DENTALIZACIÓN DE -s- EN ESPAÑOL
}

\author{
Eugenio Martínez Celdrán.
}

\section{Presentación.}

Es bien conocido el planteamiento de Navarro Tomás sobre la dentalización: "La $s$ final de sílaba, en contacto con $t[\ldots]$, toma el punto de articulación de la $t$, formándose con la punta de la lengua contra la cara interior de los incisivos superiores, y no contra los alvéolos de estos mismos dientes, como ocurre en los demás casos" ${ }^{1}$. Más adelante indica que delante de $d$ también se dentalizará, aunque su realización sea la sonora correspondiente. Después de Navarro Tomás, casi todos los autores han recogido invariablemente esta dentalización ${ }^{2}$.

Fue A. Quilis quien la puso en duda: "Desde hace tiempo, desde que empezamos a trabajar en el dominio de la fonética con el sonógrafo, veníamos dudando de la existencia real de estos dos alófonos ante $/ t, d /$, pues observábamos en los sonogramas que la frecuencia a la que aparecían las resonancias del ruido de la fricación eran iguales aproximadamente para [s] y [s]" 3. Para demostrar que efectivamente no existen esos alófonos presenta radiografías, obtenidas con el método cinerradiográfico, de las realizaciones de un solo informante madrileño. Aquí observa el autor que "aun siendo la articulación neta y claramente alveolar, la lengua tiene una inclinación que nos da ya a entender cuál va a ser la articulación que sigue" "4.

1 Manual de pronunciación española, Madrid, CSIC, § 105.

2 Cfr. R. Menéndez Pidal, Manual de gramática histórica española, Madrid, EspasaCalpe, 1968, \& 35; E. Alarcos Llorach, Fonología española, Madrid, Gredos, 1965, § 101; S. Gili Gaya, Elementos de fonética general, Madrid, Gredos, 1975, pág. 140; E. Martínez Celdrán, Fonética, Barcelona, Teide, 1989, pág. 321; M. J. Canellada y J. K. Madsen, Promunciación del español, Madrid, Castalia, 1987, pág. 36, etc.

s "Sobre los alófonos dentales de /s/", RFE, XLIX, 1966, pág. 335.

4 Ibíd., pág. 338. 
A continuación, se basará en espectrogramas de tres informantes para acabar de redondear su negación de la dentalización de $s$ en español, al no haber diferencias significativas en el comienzo del ruido de la $s$ en sus distintas realizaciones.

Recientemente hemos adquirido en nuestro Laboratorio un Electropalatógrafo. En pruebas iniciales observamos que sí había algunas diferencias destacables en los palatogramas de una $s$ anterior a $t$ en comparación con una $s$ intervocálica; por tanto, decidimos replantear la cuestión examinando los argumentos de A. Quilis y aportar nuevas pruebas basadas en otro instrumento. Como después argumentaremos, el punto de vista acústico es más complejo de lo que Quilis plantea ${ }^{5}$; pero, por otra parte, es posible que no existan diferencias de cavidad de resonancia, lo cual se refleja en una igualdad acústica. Sin embargo, el planteamiento de Navarro Tomás es meramente articulatorio, en una zona tan estrecha que una pequeña variación articulatoria puede no tener efectos acústicos $\mathrm{y}$, por tanto, perceptivos. No obstante, esa variación puede existir efectivamente. Por otra parte, un solo informante en el aspecto articulatorio no es suficiente para establecer un hecho experimental.

Nuestra hipótesis es que existen diferencias significativas en la articulación de la $s$ ante $/ t, d /$, respecto de la intervocálica y ante otras consonantes.

\section{MÉrodo.}

La electropalatografía o palatografía dinámica consiste en un paladar artificial con nueve filas de electrodos: tres en la zona alveolar, dos en la palatal y tres más en la velar (vid. fig. 1).

Este paladar artificial detecta los contactos de la lengua y envía las señales a un programa de ordenador que recoge a la misma vez la onda osciloscópica de lo pronunciado y la sucesión de contactos a lo largo del tiempo en todo el paladar. Por eso se habla de palatografía dinámica ${ }^{6}$, porque se recoge el movimiento articulatorio en todas sus fases, desde la intensiva a la distensiva (vid. fig. 2).

- Cfr. A. Jongman, S. E Blumstein y A. Lahiri, "Acoustic properties for dental and alveolar stop consonants: a cross-language study", Journal of Phonetics, 13, 1985, págs. 235-251.

- Vid. A. Marchal, La palatographie, Paris, Editions du CNRS, 1988, y D. Recasens, "L'anàlisi palatogràfica", Estudios de Fonética experimental, IV, Barcelona, PPU, 1990, págs. 13-26. 
RFE, LXXV, 1995 NUEVOS DATOS SOBRE LA DENTALización DE -S- EN ESPAÑoL 303

El instrumento utilizado en este estudio es el EPG 3 Electro-Palatograph, diseñado y desarrollado en el Speech Research Laboratory de la Universidad de Reading.

Este instrumento nos ofrece además de los gráficos anteriores otro que proporciona en porcentaje el número total de contactos de una realización delimitada en la señal osciloscópica (vid. fig. 3).

Si comparamos todos los gráficos anteriores con los tres siguientes (figura 4) de la secuencia [áşta], se observará una diferencia relevante en la primera linea del palatograma: según va progresando la articulación hacia la $t$ los contactos van aumentando. En [t] los contactos son totales. Tomada la primera línea de la $s$ anterior a $t$ (tramas 57-65) se ve

\section{$11 \quad 11 \quad 0 \quad 33 \quad 66 \quad 66$}

Es decir, una media de $31,17 \%$ de contacto de la lengua con el borde de los alvéolos. $\mathrm{La}[\mathrm{t}$ ] posee un $100 \%$ de contacto (véanse las tramas 66-81). La $s$ intervocálica por el contrario no posee ningún contacto.

Esa primera línea se ha revelado pertinente por varias razones. En primer lugar porque, aunque el ápice de la lengua esté unido a la cara interior de los incisivos superiores en la realización de [t], el borde está en estrecho contacto con la parte anterior de los alvéolos y la primera línea de los palatogramas representa los contactos de esa zona. En segundo lugar, porque realizadas unas pruebas iniciales con el primer informante comparando la primera línea con la segunda y tercera se comprobó que las dos últimas no tenían diferencias significativas. Por último, se hizo también una prueba forzando la pronunciación de una [s] dental y se vio que la primera línea tenía contactos, mientras que la [s] alveolar no los tenía.

Para realizar una observación sistemática de este fenómeno se realizaron palatogramas de cuatro informantes (tres varones y una mujer). A cada informante se le hizo pronunciar 20 secuencias con acentuación paroxítona. Cada secuencia repetía la misma vocal delante y detrás de -s-; -st-; -sd-, y $-s k$-. Se utilizaron las cinco vocales castellanas; por tanto eran secuencias como las siguientes: asa, ese, isi...asta, este, isti...asda...aska, eske...

No pueden ser muchos informantes porque el paladar artificial es personal e intransferible por razones anatómicas y de higiene: anatómicas, por- 
que cada paladar está confeccionado siguiendo el molde obtenido del paladar de cada persona; y de higiene, por motivos obvios. En nuestro laboratorio, cuatro personas se han sacrificado y se han mostrado predispuestas a soportar este tipo de experimentos.

A la misma vez las secuencias se grabaron en un cassette para poder realizar los espectros correspondientes en el espectrógrafo 5500 Sona-graph de Kay.

\section{Resultados.}

En la tabla siguiente recogemos, en primer lugar, las medias de contactos por informante según la 8 dentalizada y la no dentalizada. $Y$, en segundo lugar, exponemos los datos arrojados por la prueba de la $\mathbf{t}$ de Student ${ }^{7}$ que proporciona la significación de las diferencias entre ambos tipos de $\mathrm{s}$, con el grado de probabilidad de cometer error al asegurar que existen diferencias significativas.

\begin{tabular}{|c|c|c|c|c|}
\hline & INF. I & INF. II & INF. III & INF. IV \\
\hline$\left[\begin{array}{lllllllllllll}s] & \ldots & \ldots & \ldots & \ldots & \ldots & \ldots & \ldots & \ldots\end{array}\right.$ & 250 & 133 & 135 & 74 \\
\hline $\begin{array}{llllllllll} & \ldots & \ldots & \ldots & \ldots & \ldots & \ldots & \ldots & \ldots\end{array}$ & 34 & 0 & 0 & 0 \\
\hline $\begin{array}{lllllll}(\text { Student) } & \ldots & \ldots & \ldots & \ldots & \ldots\end{array}$ & 4.03 & 4.47 & 3.15 & 3.64 \\
\hline probabil) ... ... & 0.001 & 0.001 & 0.01 & 0.002 \\
\hline
\end{tabular}

\section{Discusión.}

\subsection{Los resultados confirman la hipótesis.}

Está claro, pues, que los datos basados en los palatogramas favorecen la hipótesis alternativa; es decir, sí existen diferencias significativas entre la $s$ que precede a las dentales y la $s$ intervocálica o que preceda a otra consonante no dental. $\mathrm{La} \mathbf{t}$ de Student proporciona probabilidades de cometer error inferiores al $1 \%$ en todos los casos; por tanto, se puede asegurar que las diferencias son significativas. Los datos palatográficos dan la razón a T. Navarro Tomás en el sentido de que hay un cierto grado de dentaliza-

7 Cfr. Ch. Butler, Statistics in linguistics, Oxford, Basil Blackwell, 1985, y Ch. Muller, Estadistica lingüistica, Madrid, Gredos, 1973. 
ción. Parece claro que $t$ y $d$ poseen un estrecho contacto con los alvéolos y no sólo con la cara interior de los incisivos superiores, lo cual queda manifiesto en la palatografía; y, como hemos visto, la $-s$ - en contacto con las dentales se comporta de forma semejante a ellas. Es cierto que, como decía A. Quilis, "se nota claramente una tendencia a ocupar el lugar de la articulación del sonido que le sigue" 8 , como se aprecia en el gráfico de la figura $6 \mathrm{~A}$. Pero además él indica que en el momento de la tensión es alveolar. $\mathrm{Si}$ se observa la figura $4 \mathrm{~B}$, las tramas $60-63$, por ejemplo, pertenecen totalmente a la tensión del sonido y en cambio hay contactos en esa primera fila que se ha demostrado significativa, cosa que no sucede en la figura $2 \mathrm{~A}$.

\subsection{Comparación con otras lenguas.}

Quilis ${ }^{9}$ alude al hecho de que las descripciones fonéticas de otras lenguas como el inglés, el francés, el alemán o el italiano no mencionan ningún tipo de dentalización en las realizaciones fonéticas de $s$. Eso puede ser por una de estas tres razones: primera, simplemente porque han despreciado una diferencia tan pequeña y sin relevancia alguna como esta; segunda, porque la $s$ que nosotros estudiamos es apical y la de esas otras lenguas es predorsal; como dice Navarro Tomás, "hay entre la $s$ española y la $s$ corriente en otros idiomas una diferencia importante; esta diferencia se manifiesta, principalmente, en la posición de la punta de la lengua, la cual en la $s$ española se eleva $[\ldots]$, mientras que en la $s$ común francesa, italiana, alemana, etc., la parte de la lengua que forma la articulación es esencialmente el predorso, quedando pasivo el ápice, el cual desciende a veces y se apoya más o menos contra los incisivos inferiores" 10 . Estas diferencias no las tiene en cuenta A. Quilis y, probablemente, el ápice se deja influir mucho más que el predorso en cuanto a las asimilaciones; por eso Navarro Tomás indica que existe dentalización, cosa que no hacen las fonéticas de las lenguas vecinas; tercera razón, D. Jones no podía mencionar alófonos dentales en las eses del inglés ya que la realización de $/ \mathrm{t}, \mathrm{d} /$ inglesas es alveolar $^{11}$. A Quilis ${ }^{12}$ aduce ejemplos tomados de D. Jones para mostrar que no transcribe la $s$ dental como: [ju:st] used to, [ju:zd] used; [zest] zest. En ninguno de estos casos

Artículo citado, pág. 337.

- Ibíd., pág. 341.

10 Op. cit., \& 106.

11 D. Jones (1918), An Outline of English Phonetics, Cambridge, C. U. P., 1980, págs. 141 y 144.

12 Artículo citado, pág. 341. 
hay justificación para una dentalización puesto que $/ \mathrm{t}$, d/ son alveolares; no se produce la secuencia $[s+$ dental $]$ como pretende Quilis. Aún más, Jones llega a decir respecto de $\mathbf{t}$ que "this articulation (dental) produces a very unnatural effect when used in English, especially when $\mathbf{t}$ is final, as in what wǫt" "13. Otro tanto podría decirse del alemán. Entre las lenguas románicas, A. M. Badia ${ }^{14}$ ha defendido también para el catalán la dentalización, como puede apreciarse claramente en sus palatogramas, que muestran un mayor contacto en la primera línea alveolar en las dentalizadas (fig. 7).

\subsection{La relación acústico-articulatoria.}

En realidad, todo el convencimiento de Quilis se ha basado sobre todo en el análisis acústico a partir de los sonogramas. Y aquí hay que darle la razón: desde el punto de vista acústico no existen diferencias, principalmente si uno se apoya en la frecuencia en que se inicia el ruido. Tampoco nosotros las hemos encontrado midiendo la frecuencia del pico con mayor intensidad en el espectro. Por ejemplo, en el primer informante:

$-s(t)-:$ media $=3318 \mathrm{~Hz}$

$-s(d)-:$ media $=3512 \mathrm{~Hz}$

$-s-\quad:$ media $=3320 \mathrm{~Hz}$

$-s(k)-:$ media $=3440 \mathrm{~Hz}$

Y en ningún caso existen diferencias significativas, al aplicar la prueba de la $\mathbf{t}$ de Student. Lo mismo sucede con los demás informantes.

No obstante, no está nada claro que estos indicios acústicos sean los relevantes para distinguir entre estos dos puntos de articulación. Habría que tener en cuenta también la amplitud, las transiciones formánticas, etc. ${ }^{15}$.

Por otra parte, la relación entre el parámetro articulatorio y el acústico no es lineal, ni proporcional. K. Stevens ${ }^{16}$ puso de manifiesto a través de la

\footnotetext{
13 Op. cit., pág. 142.

14 "Palatogrames de la llengua catalana", Estudios de Fonética Experimental, II, Barcelona, PPU, págs. 9-90.

${ }_{16}$ Cfr. S. J. Behrens y S. E. Blumstein, "Acoustic characteristics of English voiceless fricatives: a descriptive analysis", Journal of Phonetics, 16, 1988, págs. 295-298, $y$ "On the role of the amplitude of the fricative noise in the perception of place of articulation in voiceless fricative consonants", JASA, 84 (3), 1988, págs. 861-867; y Jongman, Blumstein y Lahiri, 1985, ya citados en nota 5 .

$16 \mathrm{~K}$. N. Stevens, "The quantal nature of speech: evidence from articulatoryacoustic data", en P. B. Denes y E. E. Davis (Eds.), Human Communication, A Unified View, New York, McGraw-Hill, 1972, págs. 51-66, y "On the quantal nature of speech", Journal of Phonetics, 17, 1989, págs. 3-45.
} 
teoría sobre los quanta este hecho: las diferencias articulatorias pueden producir mínimos o nulos efectos acústicos o, por el contrario, cambios de gran magnitud (vid. fig. 8).

Por tanto, es muy posible que la diferencia articulatoria entre dentales y alveolares, apenas posea una repercusión acústica como muestra la región III. Lo cual no significa que no exista la diferencia articulatoria. Simplemente, no será relevante ni en la perspectiva acústica ni en la perceptiva. Stevens et alii ${ }^{17}$ ya sugirieron que, aunque los rasgos fonéticos de las lenguas naturales están caracterizados en términos de propiedades invariantes, estas propiedades necesitan emerger sólo cuando el correspondiente rasgo fonético cumple una función pertinente en la lengua.

La teoría cuántica pone de manifiesto que existen regiones naturales que propician una serie de categorías fonéticas y una de esas regiones parece estar formada por la zona dentoalveolar sin divisiones marcadas en ella. En las lenguas del mundo es muy difícil que la región dentoalveolar se divida en dos para establecer distinciones pertinentes: "very few languages have phonologically contrastive pairs at the alveolar and dental places - they will have one or the other" 18 .

Todo lo expuesto trata de mostrar que una diferencia articulatoria no tiene por qué comportar una diferencia clara de tipo acústico o perceptivo, como sucede con la dentalización de la $s$ apical española, por eso mismo no se suele utilizar de forma pertinente.

\section{Conclusiones.}

Nuestra hipótesis ha sido confirmada por los datos palatográficos: existe dentalización en la $s$ ante $/ \mathrm{t}$, d/. Se trata de una pequeña diferencia articulatoria que no comporta modificaciones en el espectro acústico de modo llamativo. La dentalización es progresiva según los órganos se van aproximando a la articulación de la consonante siguiente, pero se puede afirmar que gran parte de la tensión de $s$ ya participa de la dentalización del sonido contiguo.

17 K. N. Stevens, S. J. Keyser y H. Kawasaki, "Toward a phonetic and phonological theory of redundant features", en J. S. PER Kel. y D. H. KLATT (Eds.), Invariance and variability of Speech processes, Hillsdale, N. J., Lawrence Erlbaum Associates, Inc. 1986.

18 C. Henton, P. Ladefoged e I. Maddieson, "Stops in the World's languages", Phonetica, 49, 2, 1992, pág. 95. 


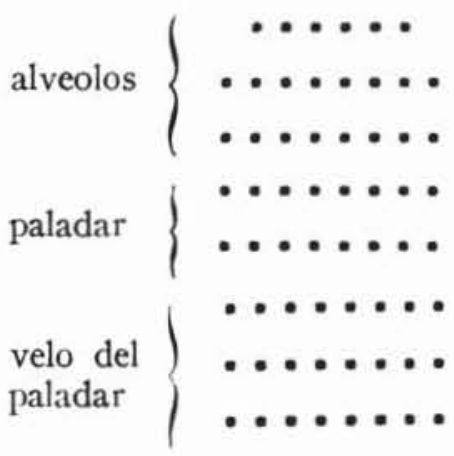

Fig. 1.-Puntos de contacto en el paladar.

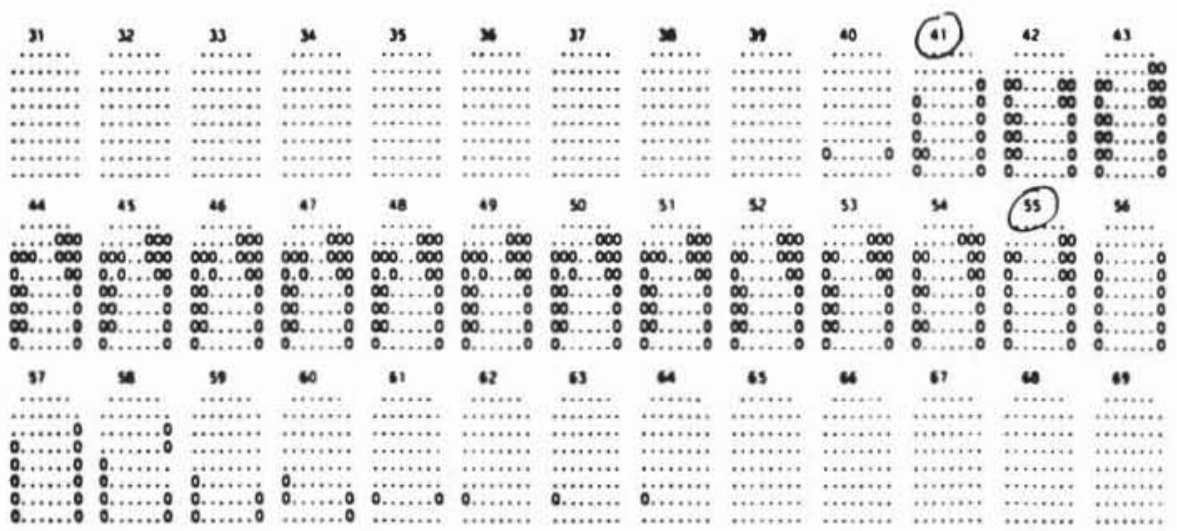

Fig. 2A-Obsérvese la sucesión de palatogramas de la secuencia [ása]. Están numerados por tramas, cada trama posee $10 \mathrm{~ms}$, la [s] se encuentra entre la trama 41 y la 55 . Los puntos de contacto de la lengua están señalados con ceros. En gran parte de la realización de [a] la lengua no tiene contacto alguno con el paladar. La $s$ sí posee contacto con los flancos del paladar.

[s]

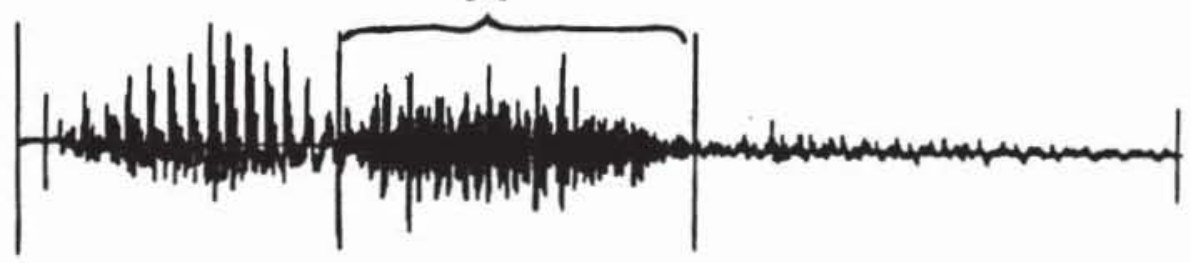

Fig. 2B.-La identificación de las tramas que ocupa un determinado sonido se realiza a través del oscilograma como se muestra en esta figura. 


$\begin{array}{rrrrrrrr} & 0 & 0 & 0 & 0 & 0 & 0 & \\ 0 & 0 & 0 & 0 & 0 & 78 & 85 & 85 \\ 92 & 92 & 57 & 0 & 0 & 71 & 92 & 100 \\ 100 & 0 & 42 & 0 & 0 & 0 & 92 & 100 \\ 100 & 92 & 0 & 0 & 0 & 0 & 0 & 100 \\ 100 & 85 & 0 & 0 & 0 & 0 & 0 & 100 \\ 100 & 100 & 0 & 0 & 0 & 0 & 0 & 100 \\ 100 & 0 & 0 & 0 & 0 & 0 & 0 & 100\end{array}$

Fig. 3.-En esta figura se ve la distribución de los contactos en la realización de [s] en la secuencia [ása], tomados entre las tramas 41 y 55 . También nos ofrece en porcentaje la frecuencia de los contactos.

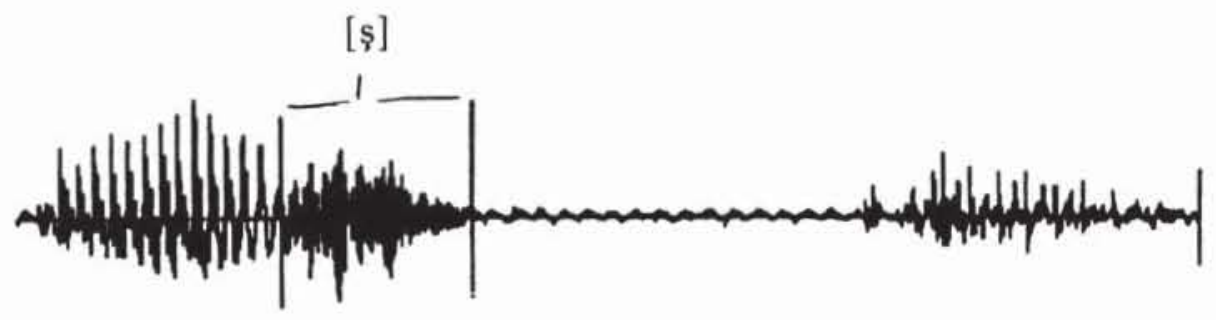

Fig. 4A,-Trazo oscilográfico de la secuencia [áş̧a] con la delimitación de la [ș].

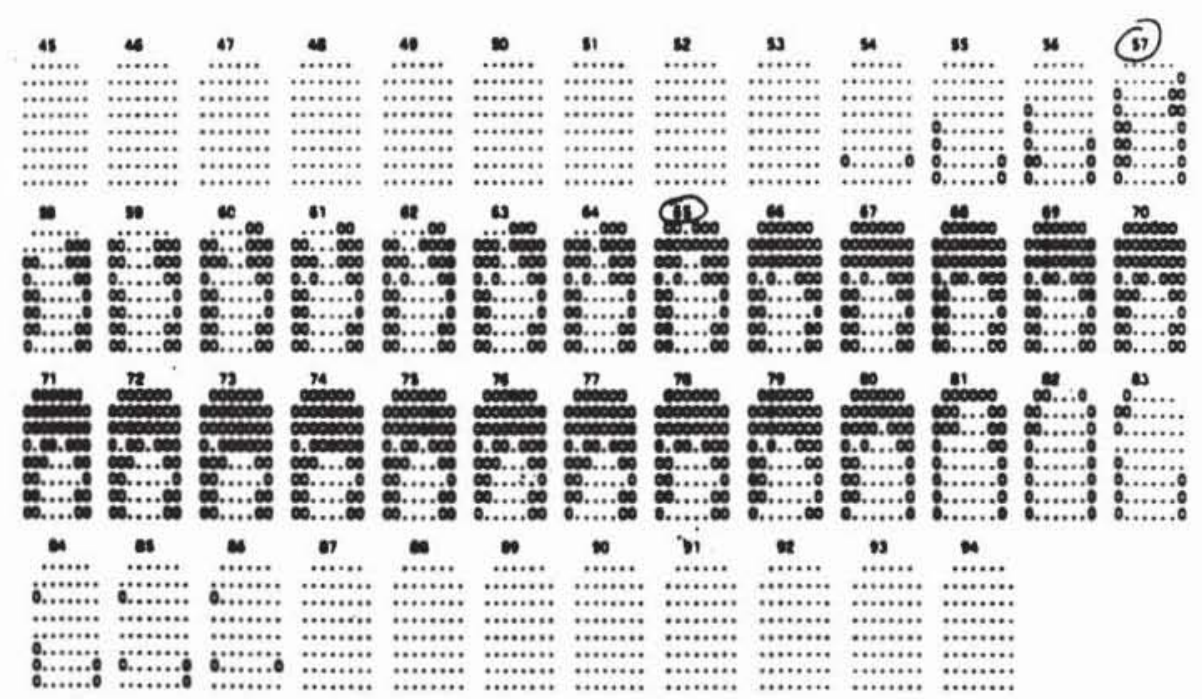

Fig. 4B.-Sucesión de palatogramas donde se ve la progresiva asimilación de $s$ a $t$. 


$\begin{array}{rrrrrrrr} & 11 & 11 & 0 & 33 & 66 & 66 \\ 77 & 77 & 33 & 11 & 44 & 88 & 88 & 100 \\ 100 & 88 & 66 & 0 & 0 & 88 & 100 & 100 \\ 100 & 0 & 55 & 0 & 0 & 22 & 100 & 100 \\ 100 & 100 & 0 & 0 & 0 & 0 & 0 & 100 \\ 100 & 100 & 0 & 0 & 0 & 0 & 0 & 100 \\ 100 & 100 & 0 & 0 & 0 & 0 & 88 & 100 \\ 100 & 77 & 0 & 0 & 0 & 0 & 88 & 100\end{array}$

Fig. 4C.-Porcentajes de contacto a lo largo de toda la realización de $s$.

$\begin{array}{lllllllll} & 0 & 0 & 0 & 0 & 70 & 94 \\ 100 & 100 & 82 & 0 & 0 & 82 & 100 & 100 \\ 100 & 100 & 100 & 0 & 5 & 88 & 100 & 100 \\ 100 & 0 & 82 & 0 & 0 & 64 & 100 & 100 \\ 100 & 100 & 0 & 0 & 0 & 0 & 100 & 100 \\ 100 & 100 & 0 & 0 & 0 & 0 & 100 & 100 \\ 100 & 100 & 0 & 0 & 0 & 5 & 100 & 100 \\ 100 & 100 & 0 & 0 & 0 & 100 & 100 & 100\end{array}$

Fig. 5.-Contactos de la realización de una [s] dental.

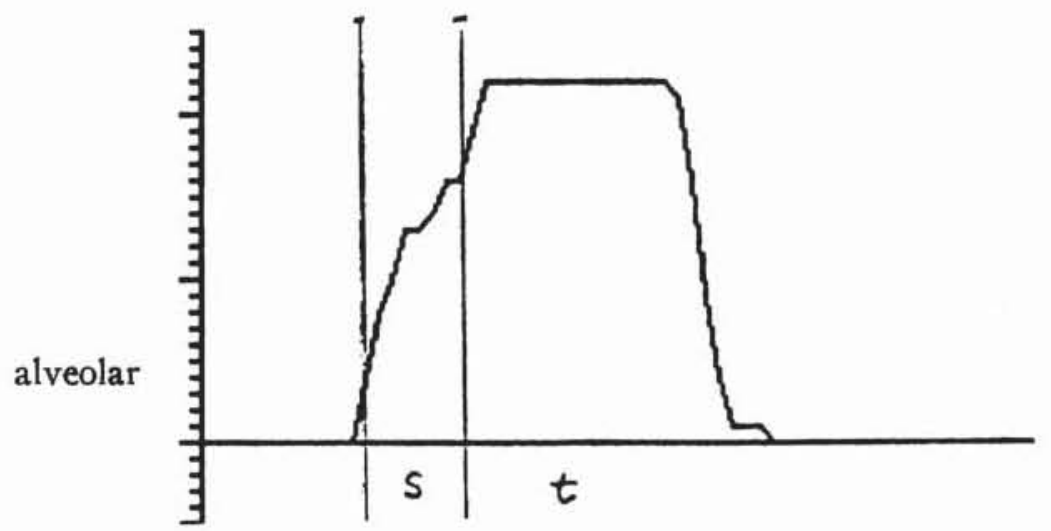

Fig. 6A.-Se observa cómo el número de contactos alveolares de la $s$ va aumentando según se aproxima a $t$ que llega al $100 \%$. 


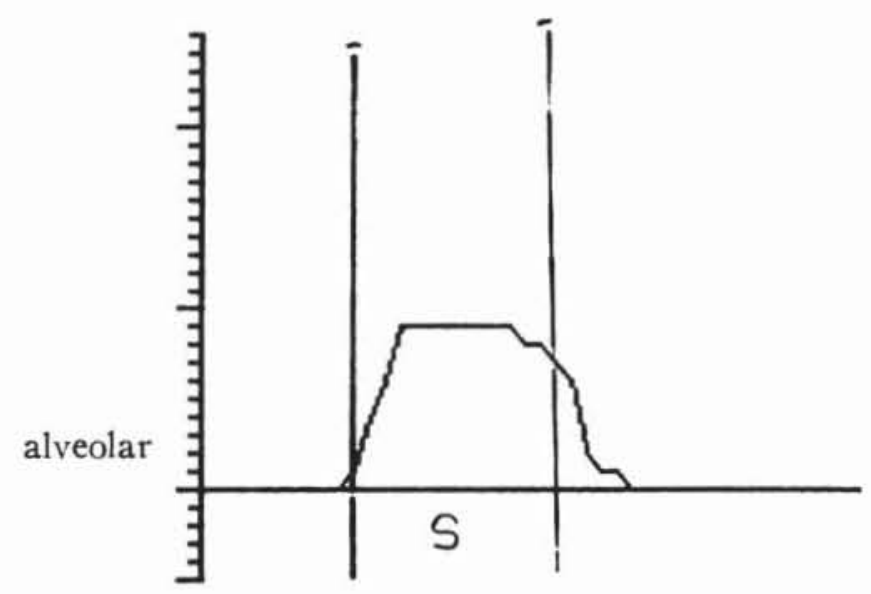

Fig. 6B.-Aquí se representa el número de contactos de $s$ intervocálica que son estables en la zona central.

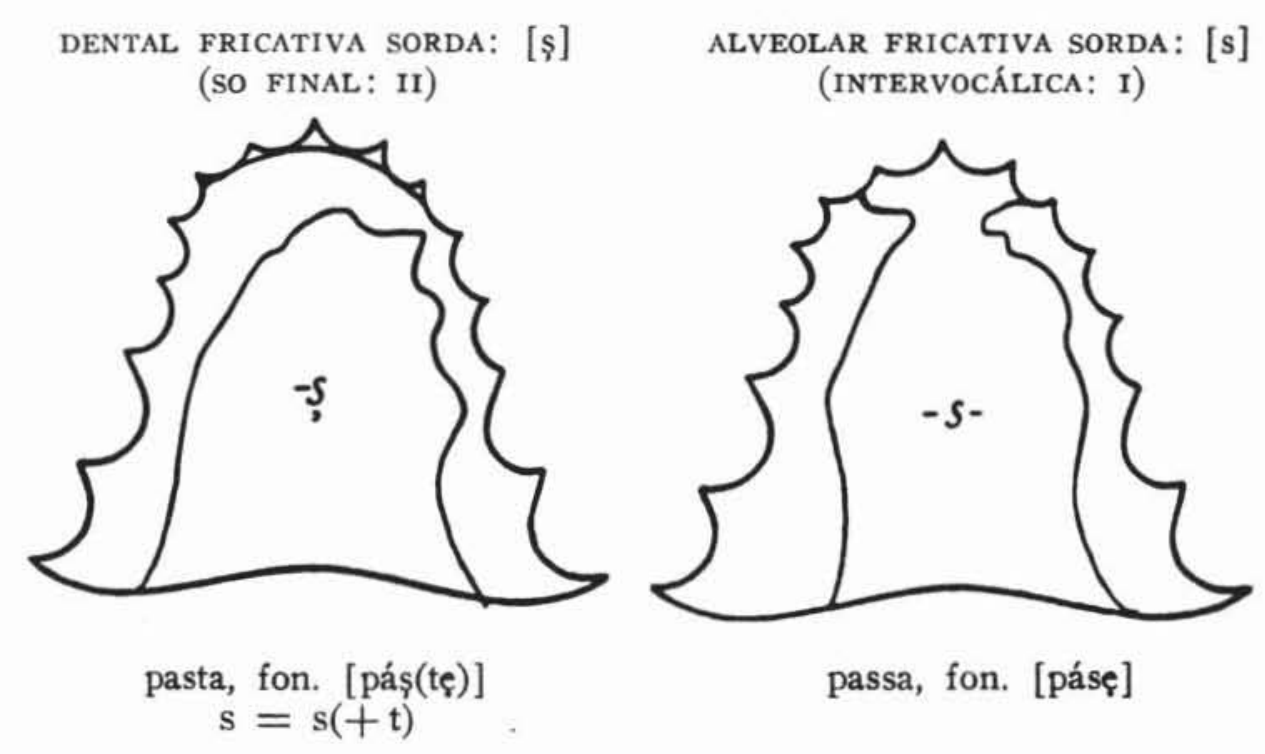

Fig. 7.-Adaptado de A. M. Badia. 


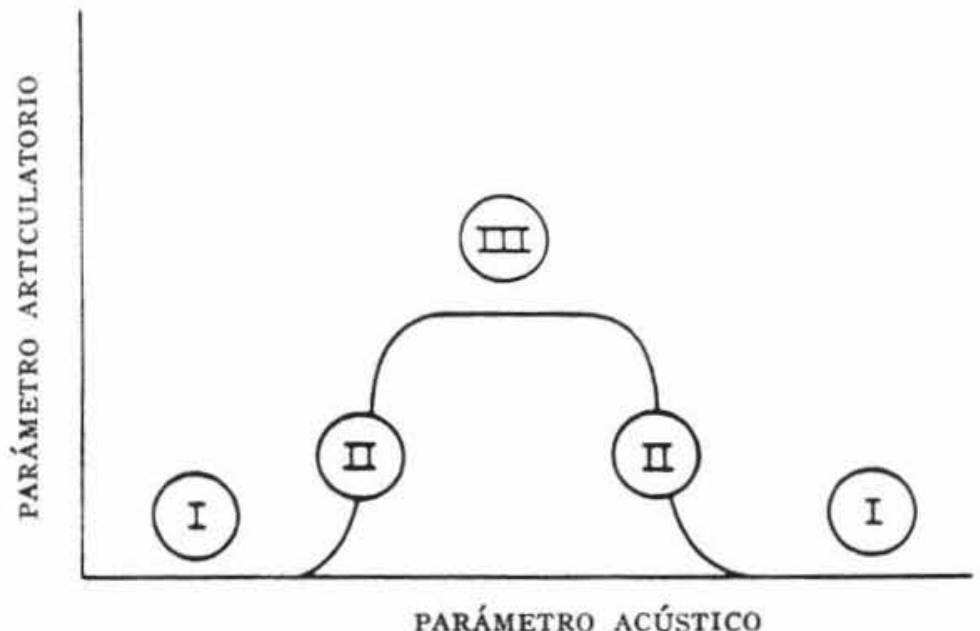

Fig. 8.-En el gráfico la región II implica que una pequeña diferencia articulatoria posee un efecto acústico considerable; en la región III se observa lo contrario: una gran diferencia articulatoria, apenas tiene diferencias acústicas. 